\title{
Lung Collapse in Intubated Patient is not Always because of Tube Factor?
}

\section{Sanjay Singhal ${ }^{1 *}$, Vivek Chopra ${ }^{2}$, Kiran $\mathrm{S}^{3}$ and Soubhadra Chakraborty ${ }^{4}$}

${ }^{1}$ Pulmonary and Critical Care Specialist, Department of Medicine, Command Hospital (CC), Lucknow 226003, India

${ }^{2}$ Classified Specialist (Anaesthesiology), Department of Anaesthesiology \& Critical Care, Command Hospital (EC), Kolkata 700027, India

${ }^{3}$ Classified Specialist (Anaesthesiology) \& Trained in Critical Care, Department of Anaesthesiology \& Critical Care, Command Hospital (EC), Kolkata 700027, India

${ }^{4}$ Post Graduate Resident (Anaesthesiology), Department of Anaesthesiology \& Critical Care, Command Hospital (EC), Kolkata 700027, India

\section{Case Summary}

50 year old male, diagnosed case of Chronic Obstructive Pulmonary Disease (COPD) was admitted to ICU as a case of cellulitis of left lower limb with septic shock and multi-organ dysfunction. On admission, he was managed with intravenous fluids, vasopressor (noradrenaline), broad spectrum antibiotics, nebulisation and mechanical ventilation. Gradually, he showed improvement clinically as well as in laboratory parameters. On fourth day, he became haemodynamically stable, maintaining oxygen saturation on minimal ventilatory support (Pressure Support mode) and was planned for extubation on the next day. But, he developed respiratory distress (respiratory rate 35/minute, use of accessory muscle present) while on Pressure Support Ventilation along with hypoxemia. He was immediately placed on control mode of mechanical ventilation with tidal volume of $350 \mathrm{ml}(6 \mathrm{mls} / \mathrm{kg})$ which showed high airway pressures ( $\mathrm{PIP}>40 \mathrm{~cm} \mathrm{H} 2 \mathrm{O}$ ) with every inspiratory effort. Suctioning of the endotracheal tube was done with a $12 \mathrm{Fr}$ Gauge suction catheter by open method which could be negotiated up to the carina with minimal secretions being aspirated with poor cough reflex. Chest auscultation revealed diminished air entry on left side. The position of the tube was re-confirmed with direct laryngoscopy. Chest radiograph revealed collapse of left lung with endotracheal tube in situ and at proper position (Figure 1). In view of whole left lung collapse and worsening hypoxemia, immediately fibreoptic bronchoscopy was done which showed thick mucus plug in the left main bronchus which after toileting was removed, following which left lung fully expanded (Figure 2)

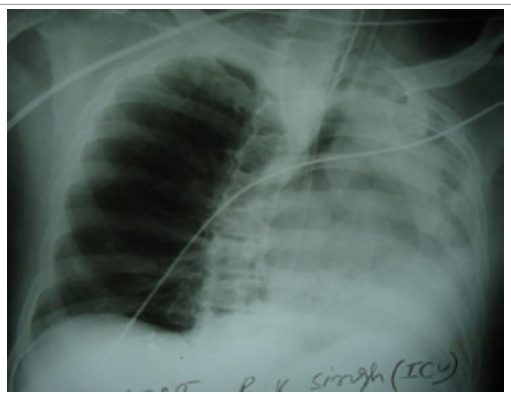

Figure 1: Chest X-ray showing atelectasis of left lung with endotracheal tube in situ.

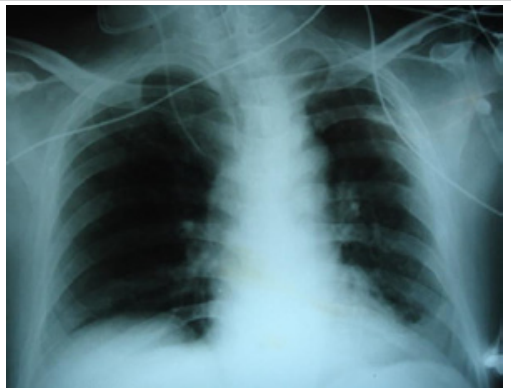

Figure 2: Chest X-ray showing complete expansion of left lung.

\section{Discussion}

Bronchial intubation on right side is common, because of the anatomy of right main bronchus, which may lead to acute left lung collapse. However, other reasons for acute lung collapse are kinking of tube, obstruction due to mucus plug, blood clot, or some foreign body. Acute lung collapse is not always due to tube factor but may be because of airway also. Significant lung collapse due to large mucus plugs occluding major airways have been described in many lung diseases such as cystic fibrosis, bronchiectasis, allergic bronchopulmonary aspergillosis, bronchial asthma and COPD [1]. Severe mucus plugging may lead to the worsening of gas exchange, increasing inspiratory pressure and difficulty in breathing; and if not treated quickly, may lead to infectious complications and mortality [2].

Maintaining good hydration, humidification of inspired gases, chest physiotherapy and postural drainage combined with manual lung hyperinflation is considered a traditional and safe method for the prevention and treatment of atelectasis in the majority of patients $[3,4]$. Although studies have shown that chest physiotherapy alone is effective for the management of acute lobar atelectasis [5]. Still, Fibreoptic bronchoscopy is a useful tool which is both diagnostic as well as therapeutic in the emergency setting (our patient was having whole lung collapse with life threatening hypoxemia) [6].

\section{References}

1. Nair SR, Pearson SB (2002) Images in clinical medicine. Mucous plug in the bronchus causing lung collapse. N Engl J Med 347: 1079.

2. Malecki S (1996) Is bronchoscopy indicated in cases of minimal tuberculosis and how it can contribute to the determination of the activity of the tuberculous process. Gruzlica 34: 265-267.

3. Konrad F, Schreiber T, Brecht-Kraus D, Georgieff M (1994) Mucociliary transport in ICU patients. Chest 105: 237-241.

4. Marini JJ, Pierson DJ, Hudson LD (1979) Acute lobar atelectasis: a prospective comparison of fiberoptic bronchoscopy and respiratory therapy. Am Rev Respir Dis 119: 971-978.

5. Gosselink R, Bott J, Johnson M, Dean E, Nava S, et al. (2008) Physiotherapy for adult patients with critical illness: recommendations of the European Respiratory Society and European Society of Intensive Care Medicine Task Force on Physiotherapy for Critically III Patients. Intensive Care Med 34: 11881199.

6. Kreider ME, Lipson DA (2003) Bronchoscopy for atelectasis in the ICU: a case report and review of the literature. Chest 124: 344-350.

*Corresponding author: Dr. Sanjay Singhal, Pulmonary and Critical Care Specialist, Command Hospital (Central Command), Lucknow 226003, India, Tel: 09335720443; E-mail: drsanjaysinghal79@yahoo.co.in

Received July 06, 2012; Accepted August 06, 2012; Published August 08, 2012

Citation: Singhal S, Chopra V, Kiran S, Chakraborty S (2012) Lung Collapse in Intubated Patient is not Always because of Tube Factor? J Clin Case Rep 2:181. doi:10.4172/2165-7920.1000181

Copyright: @ 2012 Singhal S, et al. This is an open-access article distributed under the terms of the Creative Commons Attribution License, which permits unrestricted use, distribution, and reproduction in any medium, provided the original author and source are credited. 